\title{
Solution of an Open Problem about Two Families of Orthogonal Polynomials
}

\author{
Walter VAN ASSCHE
}

Department of Mathematics, KU Leuven, Belgium

E-mail: walter.vanassche@kuleuven.be

URL: https://wis.kuleuven.be/analyse/members/walter

Received January 18, 2019; Published online January 27, 2019

https://doi.org/10.3842/SIGMA.2019.005

\begin{abstract}
An open problem about two new families of orthogonal polynomials was posed by Alhaidari. Here we will identify one of them as Wilson polynomials. The other family seems to be new but we show that they are discrete orthogonal polynomials on a bounded countable set with one accumulation point at 0 and we give some asymptotics as the degree tends to infinity.
\end{abstract}

Key words: orthogonal polynomials; special functions; open problems

2010 Mathematics Subject Classification: 42C05; 33A45

\section{Introduction}

Abdulaziz D. Alhaidari [4] submitted the following open problem for the proceedings of the OPSFA-14 conference.

Using an algebraic method for solving the wave equation in quantum mechanics, Alhaidari and co-authors $[1,2,5,6,7]$ encountered two families of orthogonal polynomials on the real line. These polynomials are defined by their three-term recurrence relations and initial values. The weight functions (orthogonality measures), generating functions, orthogonality relations, etc. are yet to be derived analytically. See [3] for more physical background.

The first family is a four parameter family of orthogonal polynomials $H_{n}^{(\mu, \nu)}(z ; \alpha, \theta)$ given by the recursion

$$
\begin{aligned}
\cos \theta & H_{n}^{(\mu, \nu)}(z ; \alpha, \theta) \\
= & \left(z \sin \theta\left[\left(n+\frac{\mu+\nu+1}{2}\right)^{2}+\alpha\right]+\frac{\nu^{2}-\mu^{2}}{(2 n+\mu+\nu)(2 n+\mu+\nu+2)}\right) H_{n}^{(\mu, \nu)}(z ; \alpha, \theta) \\
& +\frac{2(n+\mu)(n+\nu)}{(2 n+\mu+\nu)(2 n+\mu+\nu+1)} H_{n-1}^{(\mu, \nu)}(z ; \alpha, \theta) \\
& +\frac{2(n+1)(n+\mu+\nu+1)}{(2 n+\mu+\nu+1)(2 n+\mu+\nu+2)} H_{n+1}^{(\mu, \nu)}(z ; \alpha, \theta),
\end{aligned}
$$

with $0 \leq \theta \leq \pi, \mu, \nu>-1$ and $\alpha \in \mathbb{R}$. The initial values are $H_{0}^{(\mu, \nu)}(z ; \alpha, \theta)=1$ and $H_{-1}^{(\mu, \nu)}(z ; \alpha, \theta)=0$.

The second family is a three-parameter family of orthogonal polynomials $G_{n}^{(\mu, \nu)}(z ; \sigma)$ which satisfies the recurrence

$$
z G_{n}^{(\mu, \nu)}(z ; \sigma)=\left(\left(\sigma+B_{n}^{2}\right)\left[\frac{\mu^{2}-\nu^{2}}{(2 n+\mu+\nu)(2 n+\mu+\nu+2)}+1\right]\right.
$$

This paper is a contribution to the Special Issue on Orthogonal Polynomials, Special Functions and Applications (OPSFA14). The full collection is available at https://www.emis.de/journals/SIGMA/OPSFA2017.html 


$$
\begin{aligned}
& \left.-\frac{2 n(n+\nu)}{2 n+\mu+\nu}-\frac{(\mu+1)^{2}}{2}\right) G_{n}^{(\mu, \nu)}(z ; \sigma) \\
& -\left(\sigma+B_{n-1}^{2}\right) \frac{2(n+\mu)(n+\nu)}{(2 n+\mu+\nu)(2 n+\mu+\nu+1)} G_{n-1}^{(\mu, \nu)}(z ; \sigma) \\
& -\left(\sigma+B_{n}^{2}\right) \frac{2(n+1)(n+\mu+\nu+1)}{(2 n+\mu+\nu+1)(2 n+\mu+\nu+2)} G_{n+1}^{(\mu, \nu)}(z ; \sigma),
\end{aligned}
$$

with $B_{n}=n+1+\frac{\mu+\nu}{2}, \mu, \nu>-1$ and $\sigma \in \mathbb{R}$ and initial values $G_{0}^{(\mu, \nu)}(z ; \sigma)=1, G_{-1}^{(\mu, \nu)}(z ; \sigma)=0$.

Open Problem. Due to the significance of the two families $H_{n}^{(\mu, \nu)}(z ; \alpha, \theta)$ and $G_{n}^{(\mu, \nu)}(z ; \sigma)$, we hope that experts in the field of orthogonal polynomials could study them, derive their analytic properties and write them in closed form (e.g., in terms of hypergeometric functions). The required properties for these polynomials include the weight functions, generating functions, asymptotics, orthogonality relations, Rodrigues-type formulas, forward/backward shift operators, zeros, etc.

In order to identify the two families, it is convenient to switch to the monic polynomials and to look at the recurrence relation for the monic polynomials. One can then try to identify them with known families of orthogonal polynomials, e.g., by using the table of recurrence formulas in Chihara's book [8], or by using the information of hypergeometric orthogonal polynomials in the book of Koekoek, Lesky and Swarttouw [9], going through Chapter 18 on Orthogonal Polynomials in the Digital Library of Mathematical Function $[12,13]$, or by comparing with the information available in $\mathrm{CAOP}^{1}$ (Computer Algebra \& Orthogonal Polynomials). One can also try to use computer algebra to identify the polynomials from their recurrence relations, such as rec2ortho ${ }^{2}$ of Koornwinder and Swarttouw or retode ${ }^{3}$ of Koepf and Schmersau [10]. These programs were designed for older versions of maple and do not handle all orthogonal polynomials in the Askey table. Recently Tcheutia [14] extended the Maple implementation of retode to cover classical orthogonal polynomials on quadratic and q-quadratic lattices and was able to identify the second family (1.2) as Wilson polynomials, confirming the analysis in Section 3.

\section{The first family of orthogonal polynomials}

For the first family of orthogonal polynomials, the monic polynomials are given by $P_{n}(z)=$ $H_{n}^{(\mu, \nu)}(z ; \alpha, \theta) / k_{n}$, where

$$
\frac{k_{n+1}}{k_{n}}=-\frac{\sin \theta\left[\left(n+\frac{\mu+\nu+1}{2}\right)^{2}+\alpha\right](2 n+\mu+\nu+1)(2 n+\mu+\nu+2)}{2(n+1)(n+\mu+\nu+1)} .
$$

The recurrence relation then becomes

$$
z P_{n}(z)=P_{n+1}(z)+b_{n} P_{n}(z)+a_{n}^{2} P_{n-1}(z)
$$

with recurrence coefficients

$$
a_{n}^{2}=\frac{4 n(n+\mu)(n+\nu)(n+\mu+\nu)}{\sin ^{2} \theta\left[\left(n+\frac{\mu+\nu+1}{2}\right)^{2}+\alpha\right]\left[\left(n+\frac{\mu+\nu-1}{2}\right)^{2}+\alpha\right](2 n+\mu+\nu+1)(2 n+\mu+\nu)^{2}(2 n+\mu+\nu-1)}
$$

\footnotetext{
${ }^{1}$ http://www . caop.org/

${ }^{2}$ https://staff.fnwi.uva.nl/t.h.koornwinder/art/software/rec2ortho/

${ }^{3}$ http://www.mathematik. uni-kassel.de/ koepf/Publikationen/\#down
} 
and

$$
b_{n}=\frac{1}{\sin \theta\left[\left(n+\frac{\mu+\nu+1}{2}\right)^{2}+\alpha\right]}\left(\cos \theta+\frac{\mu^{2}-\nu^{2}}{(2 n+\mu+\nu)(2 n+\mu+\nu+2)}\right) .
$$

From this we can already deduce that for $\theta=\pi / 2$

$$
\lim _{\alpha \rightarrow \infty} \alpha^{n} P_{n}(z / \alpha)=P_{n}^{(\mu, \nu)}(z),
$$

where $P_{n}^{(\mu, \nu)}$ are the monic Jacobi polynomials. This follows by taking the limit for $\alpha \rightarrow \infty$ in the recurrence coefficients which, after appropriate scaling, gives the recurrence coefficients of the Jacobi polynomials. Another useful observation is that

$$
\lim _{n \rightarrow \infty} a_{n}^{2}=0, \quad \lim _{n \rightarrow \infty} b_{n}=0,
$$

and in fact $a_{n}^{2}=\mathcal{O}\left(1 / n^{4}\right)$ and $b_{n}=\mathcal{O}\left(1 / n^{2}\right)$. This implies that the Jacobi matrix

$$
J=\left(\begin{array}{cccccc}
b_{0} & a_{1} & 0 & 0 & 0 & \ldots \\
a_{1} & b_{1} & a_{2} & 0 & 0 & \ldots \\
0 & a_{2} & b_{2} & a_{3} & 0 & \ldots \\
0 & 0 & a_{3} & b_{3} & a_{4} & \\
\vdots & \vdots & & \ddots & \ddots & \ddots
\end{array}\right)
$$

is a compact operator, and in fact it is a trace class operator. This implies that the spectrum of $J$ is a countable set $\left\{x_{k}, k \in \mathbb{N}\right\}$ with one accumulation point at 0 , hence $\lim _{k \rightarrow \infty} x_{k}=0$. The trace class condition implies that $\sum_{k=0}^{\infty}\left|x_{k}\right|$ is finite. Consequently the orthogonality measure for the first family is a discrete measure supported on this countable set. See [16] for more information on compact operators and orthogonal polynomials.

The asymptotic behavior is given by

Theorem 1. There exists an entire function $Q$ such that

$$
\lim _{n \rightarrow \infty} z^{n} P_{n}(1 / z)=Q(z),
$$

uniformly on compact subsets of $\mathbb{C}$. The function $Q$ has infinitely many zeros at the points $\left\{1 / x_{k}, k \in \mathbb{N}\right\}$.

Proof. We introduce the reversed polynomials $Q_{n}(z)=z^{n} P_{n}(1 / z)$. We have $Q_{n}(0)=1$ since the $P_{n}$ are monic polynomials. The three-term recurrence relation then becomes

$$
Q_{n}(z)=Q_{n+1}(z)+b_{n} z Q_{n}(z)+a_{n}^{2} z^{2} Q_{n-1}(z),
$$

from which we easily find

$$
Q_{k+1}(z)-Q_{k}(z)=-b_{k} z Q_{k}(z)-a_{k}^{2} z^{2} Q_{k-1}(z) .
$$

Summing from $k=0$ to $n-1$ then gives

$$
\begin{aligned}
Q_{n}(z) & =Q_{0}(z)-\sum_{k=0}^{n-1} b_{k} z Q_{k}(z)-\sum_{k=1}^{n-1} a_{k}^{2} z^{2} Q_{k-1}(z) \\
& =1-\sum_{k=0}^{n-1} b_{k} z Q_{k}(z)-\sum_{k=0}^{n-2} a_{k+1}^{2} z^{2} Q_{k}(z) .
\end{aligned}
$$


Let $K$ be a compact set in $\mathbb{C}$, then there exists an $R>0$ such that $|z| \leq R$ for $z \in K$ and from (2.3) we find

$$
\left|Q_{n}(z)\right| \leq 1+\sum_{k=0}^{n-1}\left(\left|b_{k}\right| R+a_{k+1}^{2} R^{2}\right)\left|Q_{k}(z)\right| .
$$

We can then use the discrete version of Gronwall's inequality (see [15, p. 440]) to find that

$$
\left|Q_{n}(z)\right| \leq \exp \left(\sum_{k=0}^{n-1}\left(\left|b_{k}\right| R+a_{k+1}^{2} R^{2}\right)\right),
$$

so that uniformly for $z \in K$

$$
\left|Q_{n}(z)\right| \leq M=\exp \left(R \sum_{k=0}^{\infty}\left|b_{k}\right|+R^{2} \sum_{k=0}^{\infty} a_{k+1}^{2}\right) .
$$

We can then use Lebesgue's dominated convergence theorem and take the limit $n \rightarrow \infty$ in (2.3) to find

$$
\lim _{n \rightarrow \infty} Q_{n}(z)=1-\sum_{k=0}^{\infty}\left(b_{k} z+a_{k+1}^{2} z^{2}\right) Q_{k}(z):=Q(z),
$$

and the sum converges uniformly for $z \in K$. The limit function $Q$ is therefore an entire function, and its zeros are limits of the zeros of $Q_{n}$, which in turn are $\left\{1 / x_{n, k}, 1 \leq k \leq n\right\}$, where $x_{n, k}$ are the zeros of $P_{n}$.

Theorem 1 gives the existence of an entire function $Q$ for which (2.1) holds. The formula (2.4) is not very convenient to describe the limit function, since it is in terms of the polynomials $Q_{k}$ that we are investigating. In order to find more information on $Q$, we can write

$$
Q_{n}(z)=\sum_{k=0}^{n} c_{n, k} z^{k}, \quad Q(z)=\sum_{k=0}^{\infty} c_{k} z^{k},
$$

and then $c_{n, 0}=1=c_{0}$ and

$$
c_{k}=\lim _{n \rightarrow \infty} c_{n, k} .
$$

From the recurrence relation (2.2) we find

$$
c_{n, k}=c_{n+1, k}+b_{n} c_{n, k-1}+a_{n}^{2} c_{n-1, k-2},
$$

which, in principle, allows to compute the coefficients $c_{n, k}$ recursively. The first few coefficients are

$$
c_{n, 1}=-\sum_{k=0}^{n-1} b_{k}, \quad c_{n, 2}=\sum_{k=1}^{n-1} \sum_{j=0}^{k-1} b_{k} b_{j}-\sum_{k=1}^{n-1} a_{k}^{2},
$$

from which we find

$$
c_{1}=-\sum_{k=0}^{\infty} b_{k}, \quad c_{2}=\sum_{k=1}^{\infty} \sum_{j=0}^{k-1} b_{k} b_{j}-\sum_{k=1}^{\infty} a_{k}^{2} .
$$

These sums are all absolutely convergent. Their explicit value depends on the parameters $\mu, \nu$, $\alpha, \theta$ and involves the expression

$$
\psi\left(\frac{\mu+\nu+1}{2}-\sqrt{-\alpha}\right)-\psi\left(\frac{\mu+\nu+1}{2}+\sqrt{-\alpha}\right)
$$

where $\psi(z)=\Gamma^{\prime}(z) / \Gamma(z)$ is the Psi function [12, 13, Section 5.2], so that a distinction between the cases $\alpha>0$ and $\alpha<0$ may be needed. No further analysis of the function $Q$ has been done. 


\section{The second family of orthogonal polynomials}

For the second family the monic polynomials are $P_{n}(z)=G_{n}^{(\mu, \nu)}(z ; \sigma) / k_{n}$, where

$$
\frac{k_{n}}{k_{n+1}}=-\left(\sigma+B_{n}^{2}\right) \frac{2(n+1)(n+\mu+\nu+1)}{(2 n+\mu+\nu+1)(2 n+\mu+\nu+2)} .
$$

The recurrence coefficients for the monic polynomials are then given by

$$
a_{n}^{2}=\left(\sigma+B_{n-1}^{2}\right)^{2} \frac{4 n(n+\mu)(n+\nu)(n+\nu+\mu)}{(2 n+\mu+\nu-1)(2 n+\mu+\nu)^{2}(2 n+\mu+\nu+1)},
$$

and

$$
b_{n}=\left(\sigma+B_{n}^{2}\right)\left(\frac{\mu^{2}-\nu^{2}}{(2 n+\mu+\nu)(2 n+\mu+\nu+2)}+1\right)-\frac{2 n(n+\nu)}{2 n+\mu+\nu}-\frac{1}{2}(\mu+1)^{2} .
$$

Again there is a limit transition to Jacobi polynomials:

$$
\lim _{\sigma \rightarrow \infty} \sigma^{-n} P_{n}(\sigma z)=P_{n}^{(\mu, \nu)}(z-1),
$$

where $P_{n}^{(\mu, \nu)}$ are the monic Jacobi polynomials. The recurrence coefficients have the asymptotic behavior

$$
a_{n}^{2}=\frac{1}{4} n^{4}+\mathcal{O}\left(n^{3}\right), \quad b_{n}=n^{2}+\mathcal{O}(n),
$$

so that they are unbounded. The spectrum of the Jacobi matrix (and the support of the orthogonality measure for the polynomials) is therefore unbounded. It was noted by Yutian $\mathrm{Li}$ [11] that the recurrence coefficients correspond to a special case of the Wilson polynomials $W_{n}(x ; a, b, c, d)$, which are on top of the Askey table [9, Section 9.1]. The identification is

$$
G_{n}^{(\mu ; \nu)}(z, \sigma)=\frac{W_{n}(z / 2 ; a, b, c, d)}{(a+b)_{n}(a+d)_{n}}
$$

where the parameters $a, b, c, d$ are given by

$$
a=\frac{1}{2}(\mu+1)=c, \quad b=\frac{1}{2}(\mu+1)+s, \quad d=\frac{1}{2}(\mu+1)-s,
$$

with $s=\sqrt{-\sigma}$.

\section{Acknowledgements}

WVA is supported by EOS project PRIMA 30889451 and FWO research project G.086416N.

\section{References}

[1] Alhaidari A.D., An extended class of $L^{2}$-series solutions of the wave equation, Ann. Physics 317 (2005), 152-174, arXiv:quant-ph/0409002.

[2] Alhaidari A.D., Solution of the nonrelativistic wave equation using the tridiagonal representation approach, J. Math. Phys. 58 (2017), 072104, 37 pages, arXiv:1703.01268.

[3] Alhaidari A.D., Orthogonal polynomials derived from the tridiagonal representation approach, J. Math. Phys. 59 (2018), 013503, 8 pages, arXiv:1703.04039.

[4] Alhaidari A.D., Open problem in orthogonal polynomials, arXiv:1709.06081.

[5] Alhaidari A.D., Quantum mechanics with orthogonal polynomials, arXiv:1709.07652. 
[6] Alhaidari A.D., Bahlouli H., Extending the class of solvable potentials. I. The infinite potential well with a sinusoidal bottom, J. Math. Phys. 49 (2008), 082102, 13 pages.

[7] Alhaidari A.D., Ismail M.E.H., Quantum mechanics without potential function, J. Math. Phys. 56 (2015), 072107, 19 pages, arXiv:1408.4003.

[8] Chihara T.S., An introduction to orthogonal polynomials, Mathematics and its Applications, Vol. 13, Gordon and Breach Science Publishers, New York - London - Paris, 1978.

[9] Koekoek R., Lesky P.A., Swarttouw R.F., Hypergeometric orthogonal polynomials and their $q$-analogues, Springer Monographs in Mathematics, Springer-Verlag, Berlin, 2010.

[10] Koepf W., Schmersau D., Recurrence equations and their classical orthogonal polynomial solutions, Appl. Math. Comput. 128 (2002), 303-327.

[11] Li Y., Private communication with Professor Alhaidari.

[12] Olver F.W.J., Lozier D.W., Boisvert R.F., Clark C.W. (Editors), NIST handbook of mathematical functions, Cambridge University Press, Cambridge, 2010.

[13] Olver F.W.J., Olde Daalhuis A.B., Lozier D.W., Schneider B.I., Boisvert R.F., Clark C.W., Miller B.R., Saunders B.V. (Editors), NIST digital library of mathematical functions, Release 1.0.21 of 2018-12-15, available at http://dlmf .nist.gov.

[14] Tcheutia D.D., Recurrence equations and their classical orthogonal polynomial solutions on a quadratic or q-quadratic lattice, arXiv:1901.03672.

[15] Van Assche W., Asymptotics for orthogonal polynomials and three-term recurrences, in Orthogonal Polynomials (Columbus, OH, 1989), NATO Adv. Sci. Inst. Ser. C Math. Phys. Sci., Vol. 294, Kluwer Acad. Publ., Dordrecht, 1990, 435-462.

[16] Van Assche W., Compact Jacobi matrices: from Stieltjes to Krein and $M(a, b)$, Ann. Fac. Sci. Toulouse Math. (1996), 195-215, arXiv:math.CA/9510214. 